\title{
Sparse and Optimal Wide-Area Damping Control in Power Networks
}

\author{
Florian Dörfler, Mihailo R. Jovanović, Michael Chertkov, and Francesco Bullo
}

\begin{abstract}
Inter-area oscillations in power networks are typically poorly controllable by means of local decentralized control. Recent research efforts have been aimed at developing wide-area control strategies that involve communication of remote signals. In conventional wide-area control strategies the control structure is fixed a priori typically based on modal criteria. In contrast, here we employ the recently introduced paradigm of sparsity-promoting optimal control to simultaneously identify the control structure and optimize the closedloop performance. To induce a sparse control architecture, we regularize the standard quadratic performance index with an $\ell_{1}$-penalty on the feedback matrix. The quadratic objective functions are inspired by the classic slow coherency theory and are aimed at imitating homogeneous networks without interarea oscillations. We use a compelling example to demonstrate that the proposed combination of the sparsity-promoting optimal control design with the slow coherency objective functions performs almost as well as the optimal centralized controllers.
\end{abstract}

\section{INTRODUCTION}

Large-scale power networks typically exhibit multiple electromechanical oscillations. Local oscillations refer to single generators swinging relative to the rest of the grid, whereas inter-area oscillations are associated with the dynamics of power transfers and involve groups of generators oscillating relative to each other. With the steadily growing power demand, the deployment of renewables in remote areas, and the increasing deregulation of energy markets, long-distance power transfers outpace the addition of new transmission facilities. These developments lead to a maximum use of the existing network, result in smaller stability margins, and cause inter-area modes to be ever more lightly damped. In a heavily stressed grid, poorly damped interarea modes can even become unstable. For example, the blackout of August 10, 1996, resulted from an instability of the $0.25 \mathrm{~Hz}$ mode in the Western interconnected system [1].

Local oscillations are typically damped by generator excitation control via power system stabilizers (PSSs) [2]. However, these decentralized control actions can interact in an adverse way and destabilize the overall system [3]. Sometimes inter-area modes cannot be stabilized by PSSs [4], unless sufficiently many and carefully tuned PSSs are deployed [5]-[7]. Regarding tuning of convenional PSSs, high-gain feedback

This material is based in part upon work supported by the the NSF grants IIS-0904501, CPS-1135819, and CMMI-09-27720.

Florian Dörfler and Francesco Bullo are with the Center for Control, Dynamical Systems and Computation, University of California at Santa Barbara, Santa Barbara, CA 93106. M. R. Jovanović is with the Department of Electrical and Computer Engineering, University of Minnesota, Minneapolis, MN 55455. M. Chertkov is with Theory Division \& Center for Nonlinear Studies at LANL and with New Mexico Consortium, Los Alamos, NM 87544. Email: \{dorfler, bullo\}@engineering.ucsb.edu, mihailo@umn.edu, chertkov@lanl.gov is necessary in some networks [4] whereas it destabilizes other networks [5], [6]. Even when decentralized controllers provide stability, they may result in poor performance.

In principle, all the above problems can be solved by distributed wide-area control (WAC), where locally implemented controllers make use of remote measurements and control signals. WAC is nowadays feasible thanks to recent technological advances including fast and reliable communication networks, high-bandwidth and time-stamped phasor measurement units (PMUs), and flexible AC transmission system (FACTS) devices. We refer to the surveys [8], [9] and the articles in [10] for a detailed account of technological capabilities. Many efforts have been directed towards WAC of oscillations based on robust and optimal control methods, see [10]-[16] and references therein. The chosen performance metrics include frequency domain and root-locus criteria or signal amplifications from disturbance inputs to tie line flows, inter-area angles, or machine speeds. Typically, the the controllers are designed for a priori specified sensor and actuator locations and an a priori specified sparsity pattern corresponding to a communication network. In an attempt to identify optimal sensor or actuator placements and to reduce the communication complexity and the interaction among control loops, different strategies aim at identifying few but critical control channels [16]-[20]. These strategies rely on modal perspectives and aim at maximizing geometric metrics such as modal controllability and observability residues. As a result, the control channels are typically chosen through combinatorial SISO criteria and not in an optimal way.

Another body of literature relevant to our study is optimal control subject to structural constraints, for example, a desired sparsity pattern of the feedback matrix in static state feedback design [21]. In general, control design subject to structural constraints is hard, stabilizability is not guaranteed, and optimal control formulations are not convex for arbitrary structural constraints [22]. Furthermore, in the absence of pre-specified structural constraints, most optimal control problems result in controllers that require centralized implementation. In order to overcome these limitations of decentralized optimal control, alternative strategies have been recently proposed [23]-[25] that simultaneously identify the control structure and optimize the closed-loop performance.

Here we investigate a novel approach to WAC design. We follow the sparsity-promoting optimal control approach developed in [25] and find a linear static state feedback that simultaneously optimizes a standard quadratic cost criterion and enhances a sparse control structure (see Section II). Our choice of performance criterion is inspired by the classic work [26]-[28] on slow coherency. In order to improve the average closed-loop performance, we choose a performance 
criterion that encourages the closed-loop system to imitate a homogeneous network of identical generators with no inter-area oscillations. In order to reject a specific inter-area mode, we penalize the difference in the aggregate inter-area variables (corresponding to the centers of mass of the areas). Besides the physical insight, an additional advantage of our performance criteria is that the optimal controller makes use of readily accessible state variables such as angles and frequencies. We consider a coordinated PSS design for the New England Power Grid to illustrate the utility of our approach (see Section III). This compelling example shows that, with only a single WAC link, it is possible to achieve nearly the same performance as a centralized optimal controller.

\section{Problem Setup And Theoretical Framework}

\section{A. Modeling of generation, transmission, and control}

A power network is described by the dynamics of generators, power electronics, and their control equipment as well as the algebraic power flow, generator stator, and power electronic circuit equations. Some loads are also modeled in a more detailed way by differential-algebraic equations [2]. Here, we initially consider a detailed, nonlinear, and differential-algebraic power network model of the form

$$
\begin{aligned}
\dot{x}(t) & =f(x(t), z(t), u(t), \eta(t)), \\
0 & =g(x(t), z(t), u(t), \eta(t)),
\end{aligned}
$$

where the dynamic and algebraic variables $x(t) \in \mathbb{R}^{n}$ and $z(t) \in \mathbb{R}^{s}$ constitute the state, $u(t) \in \mathbb{R}^{p}$ is the control action through either power electronics (FACTS) or generator excitation (PSS) or governor control, and $\eta(t) \in \mathbb{R}^{q}$ is a white noise signal accounting for fluctuations in generation and load or communication noise in control channels.

Next we linearize the system (1) at a stationary operating point, solve the resulting linear algebraic equations for the variable $z(t)$, and arrive at the linear state-space model

$$
\dot{x}(t)=A x(t)+B_{1} \eta(t)+B_{2} u(t),
$$

where $A \in \mathbb{R}^{n \times n}, B_{1} \in \mathbb{R}^{n \times q}$, and $B_{2} \in \mathbb{R}^{n \times p}$.

\section{B. Review of slow coherency theory}

We briefly recall the classic slow coherency theory [26][28] to obtain an insightful perspective on inter-area oscillations. Let the state variable $x$ of the power network model (1) (or its linearization (2)) be partitioned as $x=\left[\theta^{T}, \dot{\theta}^{T}, x_{\mathrm{rem}}^{T}\right]$, where $\theta, \dot{\theta} \in \mathbb{R}^{n_{\mathrm{g}}}$ are the rotor angles and frequencies of $n_{\mathrm{g}}$ synchronous generators and $x_{\text {rem }} \in \mathbb{R}^{n-2 n_{\mathrm{g}}}$ are the remaining state variables, which typically correspond to fast electrical dynamics. In the absence of higher-order generator dynamics, for purely inductive lines, and for constantcurrent loads, the power system dynamics (1) reduce to the electromechanical swing dynamics of the generators [2]:

$$
M_{i} \ddot{\theta}_{i}+D_{i} \dot{\theta}_{i}=P_{\text {red }, i}-\sum_{j=1}^{n_{\mathrm{g}}}\left|Y_{\text {red }, i j}\right| E_{i} E_{j} \sin \left(\theta_{i}-\theta_{j}\right) .
$$

Here $M_{i}$ and $D_{i}$ are the inertia and damping coefficients, $E_{i}$ is the q-axis voltage, $P_{\text {red }, i}$ is the reduced power injection, and $Y_{\text {red }}$ is the Kron-reduced admittance matrix describing

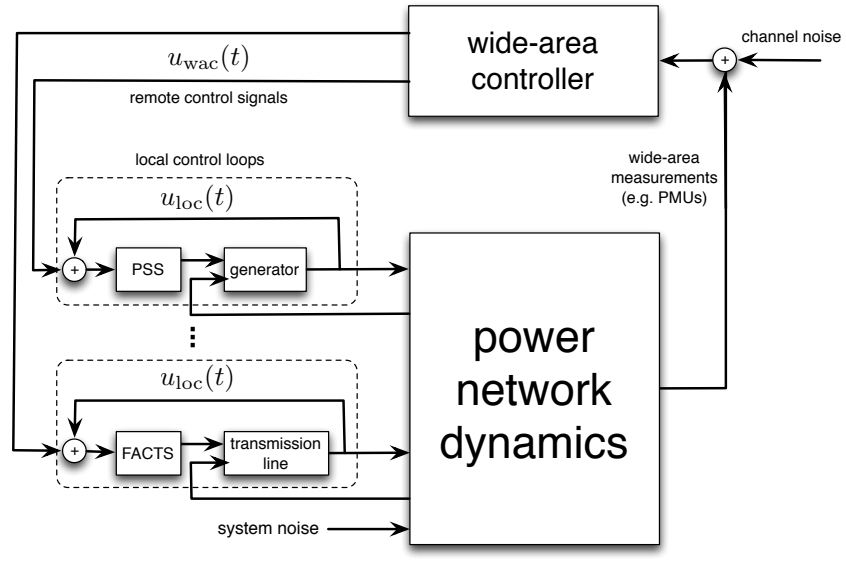

Fig. 1. Two-level control design combining local and wide-area control.

the interactions among generators. When the swing equations (3) are linearized at an operating point $\left(\dot{\theta}^{*}, \theta^{*}\right)$, they read as

$$
M \ddot{\theta}+D \dot{\theta}+L \theta=0,
$$

where $M$ and $D$ are the diagonal matrices of inertia and damping coefficients, and $L$ is the Laplacian (or admittance) matrix with off-diagonals $L_{i j}=-\left|Y_{\text {red }, i j}\right| E_{i} E_{j} \cos \left(\theta_{i}^{*}-\theta_{j}^{*}\right)$ and diagonal elements $L_{i i}=-\sum_{j=1}^{n_{\mathrm{g}}} L_{i j}$. Notice that (4) is a linear and dissipative mechanical system with kinetic energy $(1 / 2) \cdot \dot{\theta} M \dot{\theta}$ and potential energy $(1 / 2) \cdot \theta^{T} L \theta$. The mutual interactions among generators in (4) are entirely described by the weighted graph induced by the Laplacian $L$.

Inter-area oscillations arise from non-uniform inertia coefficients (resulting in slow and fast responses), clustered groups of machines (swinging coherently), and sparse interconnections among them. It can be shown [26]-[28] that the long-time dynamics of each area $\alpha$ with nodal set $\mathcal{V}_{\alpha}$ are captured by the aggregate variable $\delta_{\alpha}=$ $\left(\sum_{i \in \mathcal{V}_{\alpha}} M_{i} \theta_{i}\right) /\left(\sum_{i \in \mathcal{V}_{\alpha}} M_{i}\right)$ describing the center of mass of area $\alpha$. The slow inter-area dynamics are obtained as

$$
\tilde{M} \ddot{\delta}+\tilde{D} \dot{\delta}+\tilde{L} \delta=0,
$$

where $\delta=\left[\delta_{\alpha}, \delta_{\beta}, \ldots\right]^{T}$ and $\tilde{M}, \tilde{D}$, and $\tilde{L}$ are the reduced inertia, dissipation, and Laplacian matrices.

\section{Local and wide-area control design}

We seek for linear time-invariant control laws and follow a two-level control strategy of the form $u(t)=u_{\text {loc }}(t)+$ $u_{\text {wac }}(t)$, as illustrated in Figure 1. In a first step, the local control $u_{\text {loc }}(t)$ is designed based on locally available measurements and with the objective of stabilizing each isolated component. For example, $u_{\text {loc }}(t)$ can be obtained by a conventional PSS design with the objective to suppress local oscillations [2]. Next, the wide-area control $u_{\text {wac }}(t)$ is designed with the objective to enhance the global system behavior and to suppress inter-area oscillations. For this second design step, the local control $u_{\text {loc }}(t)$ is assumed to be absorbed into the plant dynamics (2). Due to linearity, there is no loss of generality in following this two-level strategy. Additionally, since $u_{\text {wac }}(t)$ relies on communication of remote signals, the two-level control strategy guarantees a nominal performance level in case of communication failures. 


\section{Sparsity-promoting linear quadratic control}

As discussed in Section I, an inherent problem in WAC is the proper choice of control architecture, that specifies which quantities need to be measured and which controller needs to access which measurement. Here, we confine our attention to static state feedback control laws $u_{\text {wac }}(t)=-K x(t)$, where the control structure is determined by the sparsity pattern of the feedback gain $K \in \mathbb{R}^{p \times n}$. In particular, we use the sparsity-promoting optimal control framework [25] to minimize the $\ell_{1}$-regularized steady-state variance of a stochastically driven closed-loop system:

$$
\begin{aligned}
\operatorname{minimize} & \lim _{t \rightarrow \infty} \mathcal{E}\left\{x(t)^{T} Q x(t)+u(t)^{T} R u(t)\right\} \\
& +\gamma \sum_{i, j} w_{i j}\left|K_{i j}\right|
\end{aligned}
$$

subject to

dynamics: $\quad \dot{x}(t)=A x(t)+B_{2} u(t)+B_{1} \eta(t)$,

linear control: $u(t)=-K x(t)$,

stability: $\left(A-B_{2} K\right)$ Hurwitz.

Here, $\gamma \geq 0$ is a nonnegative parameter, $\mathcal{E}\{\cdot\}$ is the expectation operator, and $Q=Q^{T} \in \mathbb{R}^{n \times n}, R=R^{T} \in \mathbb{R}^{n \times n}$ are positive semidefinite and positive definite matrices that denote the state and control weights, respectively. We assume that the triple $\left(A, B, Q^{1 / 2}\right)$ is stabilizable and detectable. The term $\sum_{i, j} w_{i j}\left|K_{i j}\right|$ is a weighted $\ell_{1}$-norm of $K$, where $w_{i j}>0$ are positive weights. The weighted $\ell_{1}$-norm serves as a proxy for the (non-convex) cardinality function $\operatorname{card}(K)$ denoting the number of non-zero entries in $K$. An effective method for enhancing sparsity is to solve a sequence of weighted $\ell_{1}$-optimization problems, where the weights are determined by the solution of the weighted $\ell_{1}$-problem in the previous iteration, see [29] for further details.

An equivalent but more versatile formulation of the optimal control problem (6) is given in terms of the feedback gain $K$ and the closed-loop observability Gramian $P$ as

$$
\begin{array}{cc}
\text { minimize } & J_{\gamma}(K) \triangleq \operatorname{trace}\left(B_{1}^{T} P B_{1}\right)+\gamma \sum_{i, j} w_{i j}\left|K_{i j}\right| \\
\text { subject to } & \left(A-B_{2} K\right)^{T} P+P\left(A-B_{2} K\right) \\
=-\left(Q+K^{T} R K\right) .
\end{array}
$$

The latter formulation (7) is amenable to an iterative solution strategy using the alternating direction method of multipliers (ADMM), see [24], [25]. The cost function in (7) is a linear combination of the $\mathcal{H}_{2}$-norm of the closed-loop system $\left(\left(A-B_{2} K\right), B_{1}, Q^{1 / 2},-R^{1 / 2} K, 0\right)$ and the sparsitypromoting term $\gamma \sum_{i, j} w_{i j}\left|K_{i j}\right|$. In what follows, for a fixed value of $\gamma \geq 0$, we denote the minimizer to (7) by $K_{\gamma}^{*}$ and the minimal cost by $J_{\gamma}^{*}=J\left(K_{\gamma}^{*}\right)$. For $\gamma=0$ the problem (7) reduces to the standard state-feedback $\mathcal{H}_{2}$-problem [30] with the optimal gain $K_{0}^{*}$ and the optimal cost $J_{0}^{*}$. On the other hand, for $\gamma>0$ the weighted $\ell_{1}$-norm promotes sparsity in the feedback gain $K_{\gamma}^{*}$, thereby identifying essential pairs of control inputs and measured outputs.

\section{E. Robustness and time delays}

A crucial objective in WAC design is robustness to time delays. Time delays may arise from communication delays, latencies and multiple data rates in the SCADA (supervisory control and data acquisition) network, and asynchronous measurements. As a result, the local control signals and measurements may have different rates and time stamps than the WAC control signals obtained from remote control or measurement sites. Standard frequency domain arguments show that robustness to such delays is directly related to phase margins [30], [31]. Since the local controllers $u_{\mathrm{loc}}(t)$ are typically designed with the objective to increase the phase $\operatorname{margin}^{1}$, and since the optimal centralized controller $K_{0}^{*}$ has a guaranteed (multivariable and non-interacting) phase margin of $\pm 60^{\circ}$, it seems plausible that $K_{\gamma}^{*}$ resulting from (7) has similar phase margins for small $\gamma>0$. Of course, these arguments are speculative since $K_{0}^{*}$ and $K_{\gamma}^{*}$ are not continuously related in $\gamma$. For our controllers, we verify robustness to delay in Section III. Alternatively, robustness to delays can be included in the design by explicitly modeling delays by Padé approximations (absorbed in the plant), or by accounting for delays via a multiplicative input uncertainty [30], [31].

\section{F. Choice of optimization objectives}

The design parameters $Q, R, B_{1}$, and $\gamma$ need to be chosen with the objective of damping inter-area oscillations. Furthermore, for the resulting feedback $u_{\mathrm{wac}}(t)=-K_{\gamma}^{*} x(t)$, the choice of control variables $K_{\gamma}^{*} x(t)$, the communication structure (the sparsity pattern of the off-diagonals of $K_{\gamma}^{*}$ ), and the control effort depend solely on $Q, R, B_{1}$, and $\gamma$.

State cost: The discussion on slow coherency theory following equation (4) implies that an ideal power system without inter-area oscillations is characterized by a homogenous interaction and uniform inertia coefficients, that is,

$L=L_{\text {unif }}=\ell \cdot\left(I_{n_{\mathrm{g}}}-\left(1 / n_{\mathrm{g}}\right) \mathbb{1}_{n_{\mathrm{g}}} \mathbb{1}_{n_{\mathrm{g}}}^{T}\right), \quad M=M_{\text {unif }}=m \cdot I_{n_{\mathrm{g}}}$, where $\ell, m>0$ are constants, $I_{n_{\mathrm{g}}}$ is the $n_{\mathrm{g}}$-dimensional identity matrix, and $\mathbb{1}_{n_{\mathrm{g}}}$ is the $n_{\mathrm{g}}$-dimensional vector of ones.

Inspired by the above considerations, we choose the following performance specifications for the state cost:

$$
x^{T} Q x=\frac{1}{2} \theta^{T} L_{\text {unif }} \theta+\frac{1}{2} \dot{\theta}^{T} M_{\text {unif }} \dot{\theta}+\varepsilon_{1} \cdot\|\theta\|_{2}^{2}+\varepsilon_{2} \cdot\|x\|_{2}^{2} .
$$

Here $\varepsilon_{1}>0$ and $\varepsilon_{2} \geq 0$ are small regularization parameters. For $\varepsilon_{1}=\varepsilon_{2}=0$, the state $\operatorname{cost} x^{T} Q x$ quantifies the kinetic and potential energy of a homogenous network composed of identical generators, and it penalizes frequency violations and angular differences, which are directly related to interarea oscillations and rotor angle instabilities. Notice also that for $\varepsilon_{1}=\varepsilon_{2}=0$, the state cost $x^{T} Q x$ does not penalize steady state deviations in the generator $d / q-$ axis voltages, the state variables of the excitation system, or the local controller $u_{\text {loc }}(t)$ included in the $A$ matrix. The additional small regularization terms $\varepsilon_{1} \cdot\|\theta\|_{2}^{2}+\varepsilon_{2} \cdot\|x\|_{2}^{2}$ are added for numerical stability and to assure detectability of $\left(A, Q^{1 / 2}\right)$.

As we will see in Section III, the state cost (8) results in an improved average closed-loop performance with all interarea modes either damped or distorted. If the objective is to reject a specific inter-area mode, then a different state cost

\footnotetext{
${ }^{1}$ For example, PSS controllers are designed to compensate for phase lags through the generator, excitation system, and power system [2].
} 
may be more appropriate. For example, if a dominant interarea mode features two groups $\mathcal{V}_{\alpha}$ and $\mathcal{V}_{\beta}$, then the discussion preceding the inter-area dynamics (5) suggests the cost

$$
\begin{aligned}
x^{T} Q x= & \ell \cdot\left\|\delta_{\alpha}-\delta_{\beta}\right\|_{2}^{2}+m \cdot\left\|\dot{\delta}_{\alpha}-\dot{\delta}_{\beta}\right\|_{2}^{2} \\
& +\varepsilon_{1} \cdot\|\theta\|_{2}^{2}+\varepsilon_{2} \cdot\|x\|_{2}^{2},
\end{aligned}
$$

where $\ell, m>0, \delta_{\alpha}, \delta_{\beta}$ are the aggregate variables, and $\varepsilon_{1}>0$ and $\varepsilon_{2} \geq 0$ are small regularization parameters. Alternative cost functions penalize certain generator frequency deviations or branch flows $\left|L_{i j}\right|\left(\theta_{i}-\theta_{j}\right)$ to assure coherency and guarantee (soft) thermal limit constraints. Finally, linear combinations of all cost functions can also be chosen.

In summary, the state costs $x^{T} Q x$ in (8) and (9) reflect the insights of slow coherency theory, they penalize only deviations from synchrony, and they are well suited objective functions for damping control. As we will see later, these choices of $Q$ also promote the use of readily available control variables, namely generator angles and frequencies.

Control cost: For simplicity and in oder to minimize interactions among generators the control effort is penalized as $u^{T} R u$, where $R$ is a positive definite and diagonal matrix.

System noise: In order to include the effects of noisy or lossy communication among spatially distributed controllers, one may choose $B_{1}=B_{2}$. Otherwise, $B_{1}$ can be chosen to include the uncertainties in load and generation in (1).

Promoting sparsity: Finally, as last degree of freedom in the optimization problem (7), we choose a sequence of $\gamma$ values. For $\gamma=0$, the problem (7) reduces to the standard optimal control problem for which a globally optimal solution can be easily obtained from the solution to the algebraic Riccati equation. Starting from this initial value, we iteratively solve the optimal control problem (7) for increasingly larger values of $\gamma$. We found that a logarithmically spaced sequence of values performs well in practice. In the end, the resulting sequence of optimal controllers is analyzed and a value of $\gamma$ is chosen that strikes a balance between the closed-loop performance and sparsity of the controller.

\section{COORDinAted SUPPLEMENTARY PSSS DESIGN}

In this section, we illustrate the WAC strategy proposed in Section II with the IEEE 39 New England power grid model consisting of 39 buses and 10 detailed two-axis generator models, where 9 generators are equipped with excitation systems and 1 generator is an equivalent aggregated model.

\section{A. Local control design and inter-area dynamics}

The Power System Toolbox [32] was used to obtain the nonlinear differential-algebraic model (1) and the linear state space system (2). The open-loop system is unstable, and the generators are equipped with PSS excitation controllers designed with washout filters and lead/lag elements. For generator $i$, the local PSS reads in the Laplace domain as

$$
u_{\mathrm{local}, i}(s)=k_{i} \cdot \frac{T_{\mathrm{w}, i} s}{1+T_{\mathrm{w}, i} s} \cdot \frac{1+T_{\mathrm{n} 1, i} s}{1+T_{\mathrm{d} 1, i} s} \cdot \frac{1+T_{\mathrm{n} 2, i} s}{1+T_{\mathrm{d} 2, i} s} \cdot \dot{\theta}_{i}(s) .
$$

The corresponding controller gains are chosen according to the optimal tuning strategy [7] as $T_{\mathrm{w}, i}=3, T_{\mathrm{n} 1, i}=T_{\mathrm{n} 2, i}=$
0.1 , and $T_{\mathrm{d} 1, i}=T_{\mathrm{d} 2, i}=0.01$ for $i \in\{1, \ldots, 10\}, k_{i}=12$ for $i \in\{1,2,3,5,6,9\}, k_{4}=10, k_{7}=11.03$, and $k_{8}=9.51$.

The local PSSs (10) with optimally tuned gains provide good damping for the local modes. An analysis of the closedloop modes and participation factors reveals the presence of four inter-area modes with relatively poor damping. These four modes are reported in Table I, and the groups of coherent machines and the frequency components of the associated eigenvectors are illustrated in Figure 2.

(a)

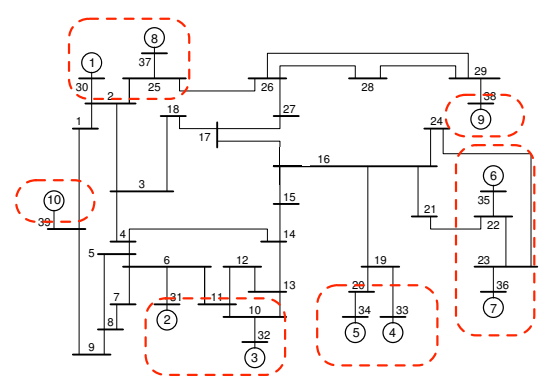

(b)

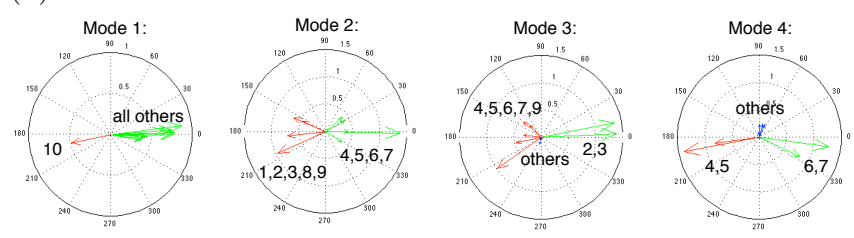

Fig. 2. Subfigure (a) displays the IEEE 39 New England power grid and its coherent groups. The polar plots in Subfigure (b) show the generator frequency components of four poorly damped inter-area modes.

TABLE I

INTER-AREA MODES OF NEW ENGLAND POWER GRID WITH PSSS

\begin{tabular}{|c|l|l|l|l|}
\hline $\begin{array}{c}\text { mode } \\
\text { no. }\end{array}$ & $\begin{array}{l}\text { eigenvalue } \\
\text { pair }\end{array}$ & $\begin{array}{l}\text { damping } \\
\text { ratio }\end{array}$ & $\begin{array}{l}\text { frequency } \\
\text { [Hz] }\end{array}$ & $\begin{array}{l}\text { coherent } \\
\text { groups }\end{array}$ \\
\hline \hline 1 & $-0.6347 \pm \mathrm{i} 3.7672$ & 0.16614 & 0.59956 & 10 vs. all others \\
2 & $-1.1310 \pm \mathrm{i} 5.7304$ & 0.19364 & 0.91202 & $1,2,3,8,9$ vs. $4-7$ \\
3 & $-1.1467 \pm \mathrm{i} 5.9095$ & 0.19049 & 0.94052 & $4,5,6,7,9$ vs. 2,3 \\
4 & $-1.5219 \pm \mathrm{i} 5.8923$ & 0.25009 & 0.93778 & 4,5 vs. 6,7 \\
\hline
\end{tabular}

\section{B. WAC design and nominal performance}

To provide additional damping for the remaining interarea modes, we design $u_{\text {wac }}(t)$ according to the sparsitypromoting optimal control problem (6), where the state cost (8) is selected with $\left(\varepsilon_{1}, \varepsilon_{2}\right)=(0.1,0)$ and gains $(\ell, m)=$ $(2,2)$. To share the control burden equally we set the control weight to be identity $R=I$. This particular choice results in a WAC signal $u_{\mathrm{wac}}(t)$ of the same magnitude as the local control signal $u_{\text {loc }}(t)$, that is, $\max _{t \in \mathbb{R}_{\geq 0}}\left\|u_{\text {wac }}(t)\right\|_{\infty} \approx$ $\max _{t \in \mathbb{R}_{\geq 0}}\left\|u_{\text {loc }}(t)\right\|_{\infty}$, and it avoids input saturation. Furthermore, to reject communication noise in the WAC implementation, we choose $B_{1}=B_{2}$. Finally, we solve the optimal control problem (7) for 40 logarithmically spaced values of $\gamma \in\left[10^{-4}, 10^{0}\right]$ and report our results in Figures 3, 4, and 5.

For $\gamma=0$, the optimal feedback gain $K_{0}^{*}$ is fully populated, thereby requiring centralized implementation. As $\gamma$ increases the off-diagonal of the feedback matrix $K$ become significantly sparser whereas the relative cost $\left(J_{\gamma}^{*}-J_{0}^{*}\right) / J_{0}^{*}$ 

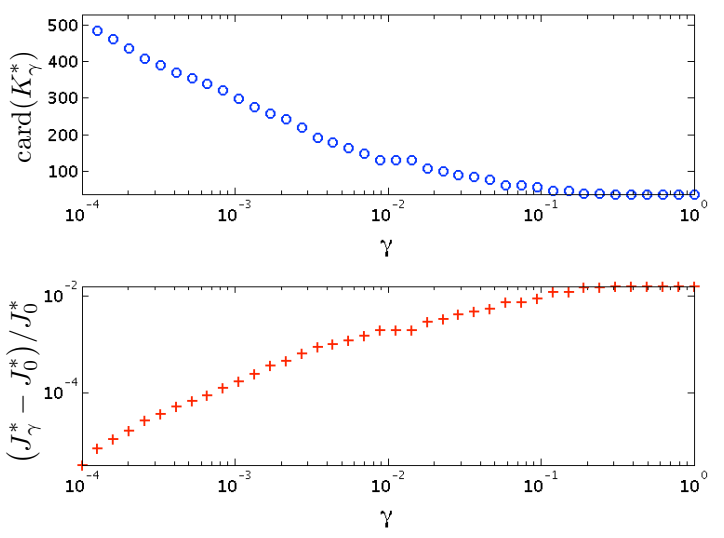

Fig. 3. Number of nonzero entries in $K_{\gamma}^{*}$ cost (relative to the cost achieved by the optimal centralized solution) $\left(J_{\gamma}^{*}-J_{0}^{*}\right) / J_{0}^{*}$ as a function of $\gamma$.

increases only slightly, see Figures 3 and 5. Additionally, as $\gamma$ increases, the state cost (8) enforces the use of angles and speeds in the off-diagonals of $K_{\gamma}^{*}$, and most nonzero elements of $K_{\gamma}^{*}$ correspond to local feedback. The final controller $K_{1}^{*}$ (for $\gamma=1$ ) is within $1.5882 \%$ of the optimal centralized performance even though only a single signal needs to be communicated: the controller at generator 1 needs to access $\theta_{9}(t)$. As expected, as $\gamma$ increases most of the control burden is on generator 1 , which has the largest inertia of all controlled generators. Likewise, the angle of loosely connected ${ }^{2}$ generator 9 needs to be measured.

The optimal feedback gain $K_{1}^{*}$ does not necessarily increase the damping of the eigenvalues associated to interarea modes. Rather, the associated eigenvectors are distorted. Here, all complex-conjugate eigenvalue pairs are left of the asymptote $\operatorname{Real}(s)=-12.74$ besides one poorly damped pair located at $-0.6229 \pm \mathrm{i} 2.4989$ possibly corresponding to the inter-area mode 1 from Table I. From the frequency components of its eigenvector in Figure 4(a) and the frequency time series in Figures 4(c) and 4(d), it can be seen that this mode does not anymore correspond to generators oscillating against each other. As a consequence, there are no more poorly damped power flow oscillations between the areas $\mathcal{V}_{\alpha}=\{1, \ldots, 9\}$ and $\mathcal{V}_{\beta}=\{10\}$; see Figures $4(\mathrm{e})$ and 4(f).

\section{Implementation issues, robustness, and delays}

The wide-area control signal can be decomposed as $u_{\text {wac }}(t)=u_{\mathrm{wac}}^{(\mathrm{loc})}(t)+u_{\mathrm{wac}}^{(\mathrm{rem})}(t)$, where $u_{\mathrm{wac}}^{(\mathrm{loc})}(t)$ corresponds to block-diagonal state feedback, which can be implemented locally using observer-based control, and $u_{\text {wac }}^{(\text {rem })}(t)$ corresponds to the remote control signal $\theta_{9}(t)$, which needs to be communicated. For our designed wide-area controller $K_{1}^{*}$, we obtain a phase margin, with respect to remote WAC control input $u_{\mathrm{wac}}^{(\mathrm{rem})}(t)$, of $89.1424^{\circ}$. The corresponding time-delay margin that can be tolerated is $27.1710 \mathrm{~s}$. We conclude that the designed controller is sufficiently robust to tolerate large communication delays, latencies in the SCADA network, and asynchronous measurements of generator rotor angles.

Additionally, we found that the information structure identified by the wide-area controller $u_{\mathrm{wac}}(t)=-K_{1}^{*} x$ is not

\footnotetext{
${ }^{2}$ In terms of graph theory, the sum of effective resistances between generator 9 and the other generators is very large compared to remaining network.
}
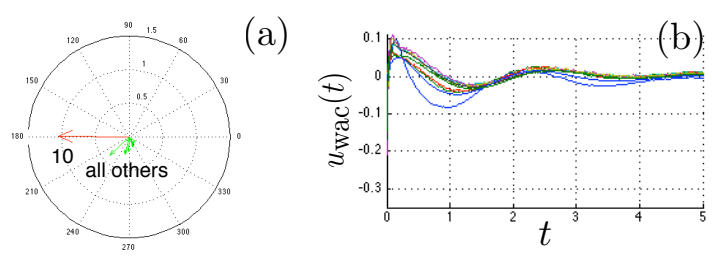

(c)
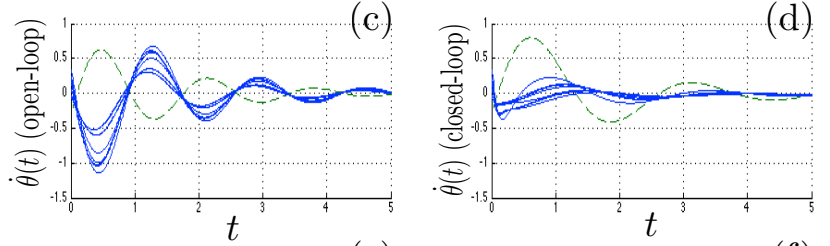

(e)
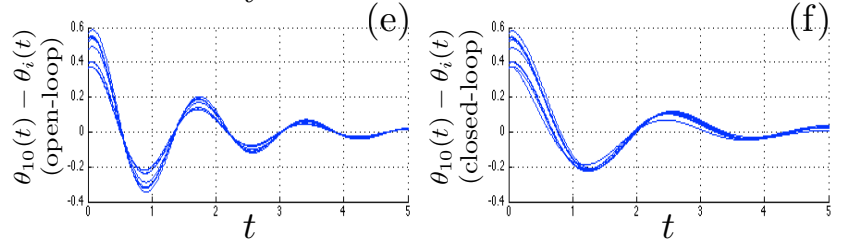

Fig. 4. Subfigure (a) displays the frequency components of the least damped oscillatory mode of the closed loop with local PSSs and WAC. Subfigure (b) shows the WAC signal $u_{\text {wac }}(t)$. Subfigures (c) and (d) depict the frequencies $\dot{\theta}(t)$ in WAC open loop (only local PSS control) and in WAC closed loop, where $\dot{\theta}_{10}(t)$ is plotted as dashed (green) curve. Subfigures (e) and (f) show the difference angles $\theta_{10}(t)-\theta_{i}(t), i \in\{1, \ldots, 9\}$, corresponding to interarea power flows in WAC open loop (only local PSS control) and in WAC closed loop. The initial conditions are aligned with the eigenvector of the dominant open-loop inter-area mode 1 , and $u_{\text {wac }}(t)$ is subject to additive white noise with zero mean and standard deviation 0.01 .

sensitive to the actual operating and linearization point in the dynamics (1). Even if the power demand is randomly altered at each load within $\pm 25 \%$ of the nominal demand (leading to different linearization matrices in (2)), the sparsity pattern of $K_{1}^{*}$ is identical to the one shown in Figure 5. We conclude that the feedback gain resulting from the sparsity-promoting optimal control problem (6) is not only characterized by low communication requirements and good closed-loop performance but also favorable robustness margins.

\section{CONCLUSIONS}

In this paper, we proposed a novel approach to WAC of inter-area oscillations. We followed a recently introduced paradigm to sparsity-promoting optimal control, and our performance objectives were inspired by the well-known slow coherency theory. We illustrated the performance of the proposed control strategy with a compelling example. Our initial results appear to be very promising, and we are currently working on extensions of the presented approach.

\section{APPENDIX}

We briefly summarize the algorithmic approach to the optimization problem (7) and refer to [25] for further details:

(i) Warm-start and homotopy: The optimal control problem (7) is solved by tracing a homotopy path that starts at the optimal centralized controller with $\gamma=0$ and continuously increasing $\gamma$ until the desired value $\gamma_{\text {des }}$ is reached;

(ii) ADMM: For each value of $\gamma \in\left[0, \gamma_{\text {des }}\right]$, the optimization problem (7) is solved iteratively using ADMM;

(iii) Updates of weights: In each step of ADMM, the weights $w_{i j}$ are updated as $w_{i j}=1 /\left(\left|K_{i j}\right|+\varepsilon\right)$ with 

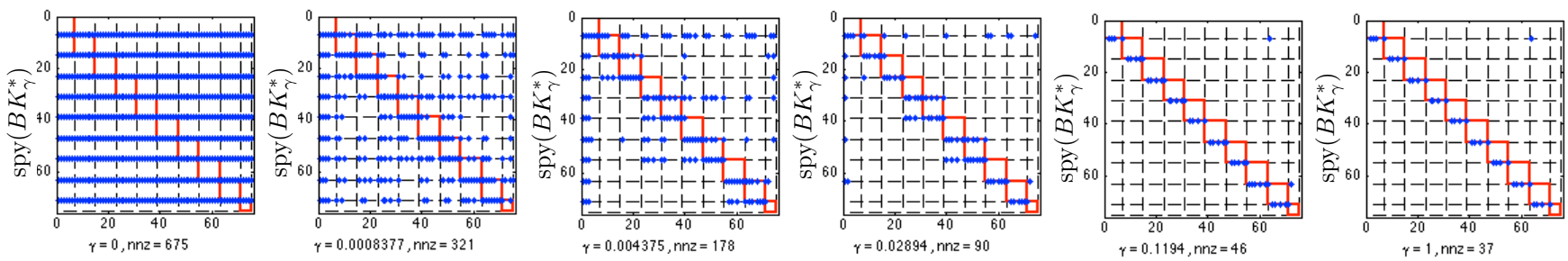

Fig. 5. The sparsity pattern of $B \cdot K_{\gamma}^{*}$ illustrates the interaction of the controllers. The different subsystems are separated in a grid, where the diagonal blocks correspond to local feedback, and the off-diagonal blocks correspond to remote feedback signals that need to be communicated. As $\gamma$ increases, the information exchange becomes sparser and angles and frequencies (the first two-states of each block) become the sole signals to be communicated.

$\varepsilon>0$. In Section III we have conducted 5 update steps with $\varepsilon=10^{-3}$; and

(iv) Polishing: Once the desired sparsity pattern $\mathcal{K}$ has been identified, the following structured optimal control problem is solved:

$$
\begin{aligned}
& \operatorname{minimize} \quad J_{\sigma}(K)=\operatorname{trace}\left(B_{1}^{T} P B_{1}\right), \\
& \text { subject to } \quad K \in \mathcal{K}, \\
& \quad\left(A-B_{2} K\right)^{T} P+P\left(A-B_{2} K\right)=-\left(Q+K^{T} R K\right) .
\end{aligned}
$$

The algorithms developed in [25] have been implemented in $M A T L A B$ and the associated software can be downloaded at www. ece. umn. edu/users/mihailo/software/lqrsp/.

\section{REFERENCES}

[1] V. Venkatasubramanian and Y. Li, "Analysis of 1996 Western American electric blackouts," in Bulk Power System Dynamics and ControlVI, Cortina d'Ampezzo, Italy, 2004.

[2] P. Kundur, Power System Stability and Control. McGraw-Hill, 1994.

[3] R. Grondin, I. Kamwa, L. Soulieres, J. Potvin, and R. Champagne, "An approach to PSS design for transient stability improvement through supplementary damping of the common low-frequency," Power Systems, IEEE Transactions on, vol. 8, no. 3, pp. 954-963, 1993.

[4] I. Kamwa, J. Beland, G. Trudel, R. Grondin, C. Lafond, and D. McNabb, "Wide-area monitoring and control at Hydro-Québec: Past, present and future," in Power Engineering Society General Meeting, 2006. IEEE. IEEE, 2006, pp. 12-pp.

[5] J. Chow, J. Sanchez-Gasca, H. Ren, and S. Wang, "Power system damping controller design-using multiple input signals," Control Systems Magazine, IEEE, vol. 20, no. 4, pp. 82-90, 2000

[6] N. Martins and L. Lima, "Eigenvalue and frequency domain analysis of small-signal electromechanical stability problems," in IEEE/PES Symposium on Applications of Eigenanalysis and Frequency Domain Methods, 1989, pp. 17-33.

[7] R. Jabr, B. Pal, N. Martins, and J. Ferraz, "Robust and coordinated tuning of power system stabiliser gains using sequential linear programming," Generation, Transmission \& Distribution, IET, vol. 4, no. 8, pp. 893-904, 2010.

[8] K. Seethalekshmi, S. Singh, and S. Srivastava, "Wide-area protection and control: Present status and key challenges," in Fifteenth National Power Systems Conference, IIT Bombay. PSCE, December 2008, pp. 169-175.

[9] J. Xiao, F. Wen, C. Chung, and K. Wong, "Wide-area protection and its applications-a bibliographical survey," in Power Systems Conference and Exposition, 2006. PSCE'06. 2006 IEEE PES. IEEE, 2006, pp. 1388-1397.

[10] M. Amin, "Special issue on energy infrastructure defense systems," Proceedings of the IEEE, vol. 93, no. 5, pp. 855 -860, may 2005.

[11] G. Boukarim, S. Wang, J. Chow, G. Taranto, and N. Martins, "A comparison of classical, robust, and decentralized control designs for multiple power system stabilizers," Power Systems, IEEE Transactions on, vol. 15, no. 4, pp. 1287-1292, 2000.

[12] D. Dotta, A. Silva, and I. Decker, "Power system small-signal angular stability enhancement using synchronized phasor measurements," in Power Engineering Society General Meeting, 2007. IEEE. IEEE, 2007, pp. $1-8$
[13] Q. Liu, V. Vittal, and N. Elia, "LPV supplementary damping controller design for a thyristor controlled series capacitor (TCSC) device," Power Systems, IEEE Transactions on, vol. 21, no. 3, pp. 1242-1249, 2006.

[14] K. Tomsovic, D. Bakken, V. Venkatasubramanian, and A. Bose, "Designing the next generation of real-time control, communication, and computations for large power systems," Proceedings of the IEEE, vol. 93, no. 5, pp. 965-979, 2005.

[15] A. Chakrabortty, "Wide-area damping control of power systems using dynamic clustering and tcsc-based redesigns," Smart Grid, IEEE Transactions on, vol. 3, no. 3, pp. 1503-1514, 2012.

[16] Y. Zhang and A. Bose, "Design of wide-area damping controllers for interarea oscillations," Power Systems, IEEE Transactions on, vol. 23 , no. 3, pp. 1136-1143, 2008

[17] A. Heniche and I. Karnwa, "Control loops selection to damp inter-area oscillations of electrical networks," Power Systems, IEEE Transactions on, vol. 17, no. 2, pp. 378-384, 2002.

[18] Y. Zhang, "Design of wide-area damping control systems for power system low-frequency inter-area oscillations," Ph.D. dissertation, Washington State University, 2007.

[19] H. Nguyen-Duc, L. Dessaint, A. Okou, and I. Kamwa, "Selection of input/output signals for wide area control loops," in Power and Energy Society General Meeting, 2010 IEEE. IEEE, 2010, pp. 1-7.

[20] L. Kunjumuhammed, R. Singh, and B. Pal, "Robust signal selection for damping of inter-area oscillations," Generation, Transmission \& Distribution, IET, vol. 6, no. 5, pp. 404-416, 2012.

[21] F. Lin, M. Fardad, and M. R. Jovanović, "Augmented Lagrangian approach to design of structured optimal state feedback gains," IEEE Trans. Automat. Control, vol. 56, no. 12, pp. 2923-2929, December 2011

[22] A. Mahajan, N. C. Martins, M. C. Rotkowitz, and S. Yuksel, "Information structures in optimal decentralized control," in IEEE Conf. on Decision and Control, Maui, HI, USA, Dec. 2012, pp. 1291 -1306.

[23] S. Schuler, U. Münz, and F. Allgöwer, "Decentralized state feedback control for interconnected process systems," in In Proc. of the 8th IFAC Symposium on Advanced Control of Chemical Processes (AdChem), Jul. 2012, pp. 1-10.

[24] F. Lin, M. Fardad, and M. R. Jovanović, "Sparse feedback synthesis via the alternating direction method of multipliers," in Proceedings of the 2012 American Control Conference, Montréal, Canada, 2012, pp. 4765-4770.

[25] — - "Design of optimal sparse feedback gains via the alternating direction method of multipliers," IEEE Trans. Automat. Control, 2013, provisionally accepted; also arXiv:1111.6188.

[26] J. H. Chow, Time-scale modeling of dynamic networks with applications to power systems. Springer, 1982.

[27] J. H. Chow and P. Kokotović, "Time scale modeling of sparse dynamic networks," IEEE Transactions on Automatic Control, vol. 30, no. 8 , pp. 714-722, 1985.

[28] D. Romeres, F. Dörfler, and F. Bullo, "Novel results on slow coherency in consensus and power networks," in European Control Conference, Zürich, Switzerland, Jul. 2013, to appear.

[29] E. Candès, M. Wakin, and S. Boyd, "Enhancing sparsity by reweighted $\ell_{1}$ minimization," Journal of Fourier Analysis and Applications, vol. 14, no. 5, pp. 877-905, 2008.

[30] G. E. Dullerud and F. Paganini, A Course in Robust Control Theory, ser. Texts in Applied Mathematics. Springer, 2000, no. 36.

[31] S. Skogestad and I. Postlethwaite, Multivariable Feedback Control Analysis and Design, 2nd ed. Wiley, 2005.

[32] J. Chow and K. Cheung, "A toolbox for power system dynamics and control engineering education and research," Power Systems, IEEE Transactions on, vol. 7, no. 4, pp. 1559-1564, 1992. 